ARTICULOS originales

\title{
Capacidad de concentración urinaria en lactantes desnutridos. I Parte
}

DRES.: JOSE CARDENAS J. **, FEderico puGa C. *, GASTON zILlERUElo B. *.

INTRODUCCION. La capacidad de concentrar la orina mediante la reabsorción a nivel de túbulo colector es de gran importancia entre las múltiples funciones del riñón, destinadas a mantener la homeostasis. Este es el mecanismo primatio por el que el organismo elimina excesos de agua o la retiene en casos de necesidad. En este proceso el túbulo colector actúa como efector a la acción de la Hormona antidiurética. Esta hormona es producida a nivel de las neuronas del núcleo supraóptico del hipotálamo y se acumula en el lóbulo posterior de la hipófisis $(1,2)$.

Es secretada en el organismo como respuesta a tres tipos de estímulos:

a) Actividad del Sistema Nervioso Central.

b) Variaciones del volumen intravascular.

c) Variaciones en la osmolaridad plasmática.

La variación en la osmolaridad plasmática es el estímulo de mayor importancia:

El descenso en la osmolaridad, frena la secreción de $H A D$, con la consiguiente disminución en la reabsorción de agua a njvel del túbulo colector. La hiperosmolaridad estimula la secreción de la hormona, produciendo reabsorción tubular de agua y descenso en la osmolaridad plasmática (3).

Esta respuesta es simultánea con la estimulación producida por variaciones de volumen intravascular.

Existen condiciones anormales, en las que esta función renal no puede llevarse a cabo. Tres grandes grupos de anomalías podrían ser señalados como causas:

\footnotetext{
* Unidad de Nefrolugía. Departamento de Pediatría. HospitaI Luis Calvo Mackenna.

** Eecado, Departameato de Pediatria. Hospital Luis Calvo Mackenna.
}

I. Defectos en la secreción y/o producción de HAD.

II. Defectos a nivel del efector renal.

III. Interacción de los grupos anteriores.

En el primer grupo, tenemos la Diabetes Insípida Neurogénica, entidad de múltiples etiologías, en las cuales el denominador común es la disfunción de la unidad del hipotâlamo hipofisiaria, reflejada en una producción insuficiente de HAD.

En el segundo grupo está la Diabetes Insípida Nefrogénica. En este Sindrome, niveles útiles de $\mathrm{HAD}$, no encuentran respuesta adecuada en el efector renal. Se invocan múltiples mecanismos y etiologías dentro de este Sindrome $(4,5,6)$.

En nuestro trabaja nos dedicaremos, en parte, al estudio de una situación que podría ser considerada como característica del tercer grupo: La Desnutrición. En ella se conjugarían los dos mecanismos.

Material $y$ Método. En 23 lactantes del Hospital Luis Calvo Mackenna, en quienes no había patología renal asociada a sus grados variables de desnutrición, se hicieron pruebas de concentración urinaria.

El método utilizado consistió en la asociación a una deprivación acuosa de 12 horas, de una dosis de vasopresina. Esta se colocó intramuscular en forma de Tanato de Pitresina (Tanato de Pitresina en suspensión oleosa, Parke Davis) en dosis de 0,08 U. vasopresoras por kilo de peso. Dos horas después se inició la deprivación acuosa, que consistió en la suspensión de toda ingesta.

Las muestras de orina para determinar la osmolaridad, se tomaron mediante recolectores, en 
períodos de tiempo de dos horas, a partir del término de la deprivación acuosa. Al inicio de la recolección de orina se tomaron muestras de sangre para medir osmolaridad y electrolitos plasmáticos.

Las osmolaridades se midieron en un osmómetro (Osmette, Precition Osmometer).

Tomándose como valores útiles aquellos de máxima concentración, cuya diferencia entre dos lecturas de la misma muestra no fuera superior a. 2 mOsm. Los electrolitos plasraáticos fueron determinados mediante un espectrofotómetro de llama.

El estado nutritivo de los niños fue determinado en base al porcentaje de déficit de peso, referido al peso ideal para su edad. Los pesos ideales fueron tomados de las "Normas de Supervisión del crecimiento y desarrollo infantil" deI Servicio Nacional de Salud de Chile, publicados en Santiago en 1972. En base a estos porcentajes se hicieron tres grapos que correspondían a nifios con déficit entre 0 y $20 ; 20$ y $40 ; 40$ y $60 \%$ respectivamente.

Resultados. Ier, Grupo: Con porcentajes de déficit de peso comprendidos entre 0 y $20 \%$, cuyas edades fluctuaban entre 8 meses y 2 años, con un promedio etario de 13,2 meses.

La osmolaridad urinaria promedio fue de $1.044 \mathrm{~m} \mathrm{Osm} / 1$ con una máxima de $1.167 \mathrm{~m}$. Osm/1 y una mínima de $957 \mathrm{~m}$ Osm/1.

La osmolaridad plasmática promedio fue de $316 \mathrm{~m} \mathrm{Osm} / 1$ con una máxima de $332 \mathrm{~m} \mathrm{Osm/1}$ y una minima de $298 \mathrm{~m}$ Osm $/ 1$.

Los electrolitos en sangre promedios fueron de $145,1 \mathrm{meq} \mathrm{Na} / 1 ; 104 \mathrm{meq} \mathrm{Cl}-/ 1$, y $4,6 \mathrm{meq}$ $\mathrm{K}^{+} / 1$. Con rangos entre 140 y 149 meq $/ 1$ para el Sodio, 108 y $97 \mathrm{meq} / 1$ para el Cloro, y 4,5 y $5,2 \mathrm{meq} / 1$ para el potasio.

$2^{\circ}$ Grupo: Con porcentajes de déficit de peso, comprendidos entre 20 y $40 \%, 9$ niños, cuyas edades variaban entre 4 y 21 meses, con una edad promedio de 10 meses.

La osmolaridad urinaria promedio fue de $917 \mathrm{~m}$ Osm $/ 1$; con $840 \mathrm{~m}$ Osm $/ 1$ de mínima y $1.004 \mathrm{~m} \mathrm{Osm} / 1$ de máxima.

La osmolaridad plasmática promedio fue de $312,7 \mathrm{~m} \mathrm{Osm} / 1$ con valores que fluctuaban entre 306 y $325 \mathrm{~m} \mathrm{Osm} / 1$.

Los valores promedios de $\mathrm{Na}^{-1}, \mathrm{Cl},-$ y Ken sangre fueron de 144,$8 ; 103,6$ y $4,9 \mathrm{meq} / 1$ respectivamente, con valores que fluctuaban entre 138 y $147 \mathrm{meq} / 1$ para el $\mathrm{Na}^{+} ; 96,3$ y $110 \mathrm{meq} / 1$ para el $\mathrm{Cl}^{-}$; y 4,5 y $5,1 \mathrm{meq} / 1$ para el $\mathrm{K}-$.

3er. Grupo: Con porcentajes de déficit de peso, comprendidos entre el 40 y $60 \%$, 6 niños, cuyas edades variaban entre 5 y 18 meses, con un promedio etario de 10,7 meses.
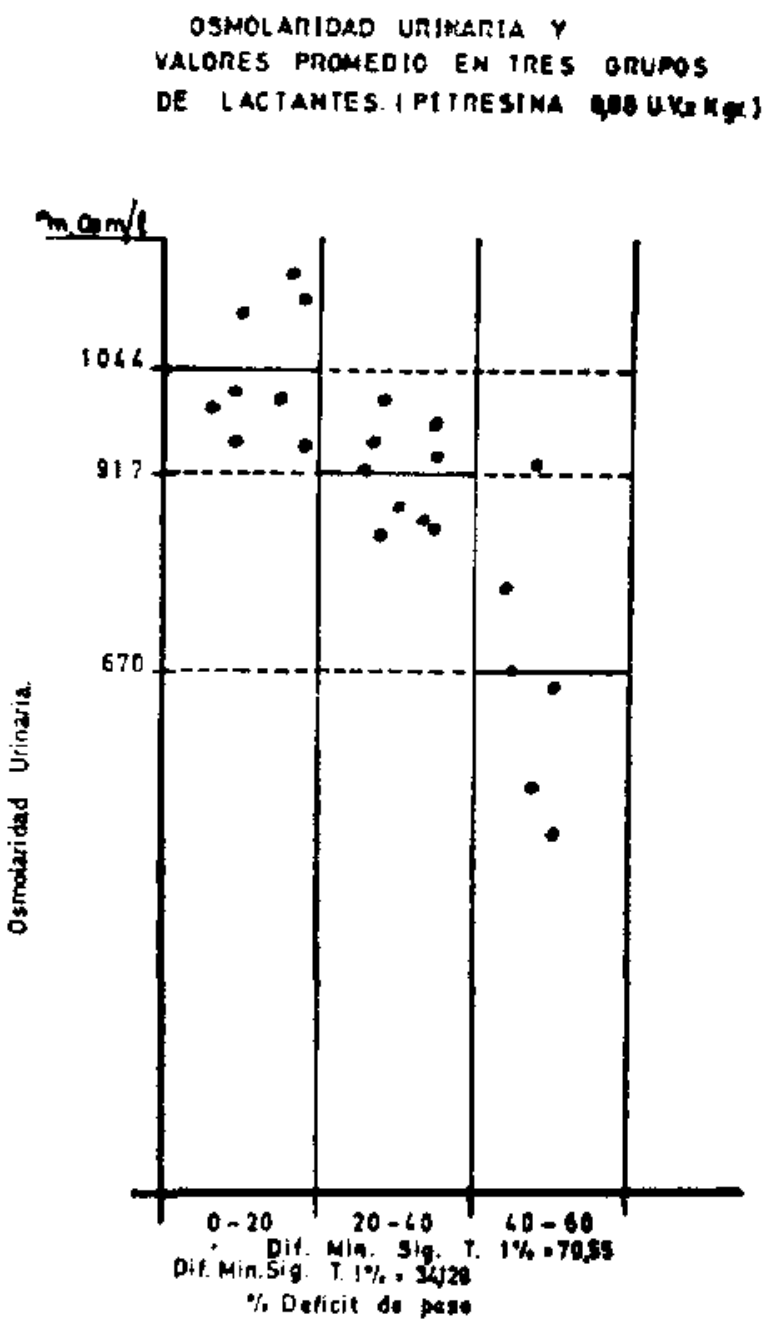

Figura No 1

La osmolaridad urinaria promedio fue de $670 \mathrm{~m} \mathrm{Osm} / 1$; con $160 \mathrm{~m} \mathrm{Osm} / 1$ de mínima y $928 \mathrm{~m} \mathrm{Osm} / 1$ de máxima.

La osmolaridad plasmática fue de $300 \mathrm{~m}$ Osm/1 con rangos de $290 \mathrm{~m}$ Osm/1 de mínima y $310 \mathrm{~m} \mathrm{Osm} / 1$ de mínima y $310 \mathrm{~m} \mathrm{Osm} / 1$ de máxima.

Los valores promedios de $\mathrm{Na}^{+}, \mathrm{Cl}-, \mathrm{y} \mathrm{K}^{+}$ en sangre fueron de $144 \mathrm{meq} / 1 ; 104 \mathrm{meq} / 1 \mathrm{y}$ $4,8 \mathrm{meq} / 1$ respectivamente, con valores que variaban entre 137 y $150 \mathrm{meq} / 1$ para el $\mathrm{Na}, 100 \mathrm{y}$ $108 \mathrm{meq} / 1$ para el Cloro y 4,0 y $5 \mathrm{meq} / 1$ para el Potasio (Ver figura 1).

COMEnTARIos. La capacidad del riñón para concentrar la orina puede sufrir alteraciones de diversas etiologías, como dijimos al iniciar este trabajo.

En el desnutrido este fenómeno ha sido estudiado por algunos autores. 
Gordillo (8) y Alleyne (9) han hecho una evaluación intensiva de la función renal en general y atribuyen la incapacidad para concentrar la orina, en estos niños a una ruptura en la unidad "túbulo-medular", con disminución del gradiente osmolar, causada por una hipotonía a nivel del intersticio medular. Varias serían también las causas de esta ruptura; entre ellas las más importantes son:

1. Descenso en la Velocidad de Filtación Glomerular (V. F. G.) $(8,9)$.

2. Flujo Plasmático Renal (F., P. R.) disminuído (9).

3. Baja concentración plasmática de Sodio y Urea. $(10,11,12)$.

4. Baja concentración de $\mathrm{K}^{\lrcorner} \mathrm{y} \mathrm{Mg}^{++}(13,14$, 15).

Algunos de estos factores incidirían directamente en la formación de una hipotonía a nivel de intersticio medular, por ejemplo: la Hiponatremia y los niveles bajos de urea plasmática.

Los otros to harían en forma indirecta a través de una disminución en la VFG, lo que como ha sido demostrado (16), altera la capacidad de concentración urinaria, debido a que probablemente disminuye el aporte de $\mathrm{Na}^{+}$y urea a la médula renal, lo que limitaría el establecimiento de una gradiente osmótica efectiva.

Gordillo (17) menciona la posibilidad de que además de los factores antes enumerados. existiría ưna disminución en los niveles de HAD, - una falla en la respuesta tubular a dicha hormona, en especial, en aquellos desnutridos con Nefropatía Kaliopénica (18). Vial y Donoso (19) consideran de que además de trastornos meduJares podría haber alteraciones metabólicas a nive] de las células tubulares.

En nuestra experiencia, obscrvamos que con dosis de pitresina de $0,08 \mathrm{UV} \times \mathrm{Kgs}$. de peso $\mathrm{y}$ 12 horas de deprivación acuosa, existía una diferencia estadísticamente significativa entre los promedios de osmolaridad urinaria obtenidos en los tres grupos de lactantes.

Fue en el grupo con desnutrición más intensa $(40-60 \%)$, el que alcanzó el nivel más bajo $(670 \mathrm{~m} \mathrm{Osm} / 1)$, en tanto que el grupo formado por niños eutróficos o con desnutrición leve $(0-20 \%)$ mostró niveles comparables a los encontrados en escolares, sin alteración aparente en la función renal. Debemos hacer notar que aunque los análisis estadísticos validan nuestros resultados, la muestra estudiada es reducida, por to que consideramos convenientc realizar estudios en grupos más extensos.

El hecho evidente en nuestra experiencia, do que la incapacidad renal para concentrar la orina en el desnutrido es proporcional al grado de desnutrición, nos permite hacer una serie de consideraciones para tratar de explicarnos este fenómeno.

La presencia di niveles de $\mathrm{Na}^{--}, \mathrm{Cl}^{-}$y $\mathrm{K}^{+}$ plasmáticos normales en todos los grupos, en el momento de realizar la prueba, nos permite descartar un déficit de electrolitos como factor desencadenante de esta falla. Aunque no se midió niveles de urea en sangre ni orina, el hecho que existieran niveles de osmolaridad plasmática baja en grupos con desnutrición severa, nos hace pensar que existía niveles disminuídos de urea plasmática en nuestros pacientes, hecho que condicionaría la incadacidad para concentrar. Esto estaría en contradicción con las conclusiones obtenidas por Edelmann y Barnett (20) en su trabajo, quienes atribuyen a la urea un papel insignificante en los mecanismos de concentración urinaria del lactante. No existiría esta discordancia, si hubiese en el desnutrido otro factor más importante que condicione esta disfunción. Nosotros consideramos la posibilidad de que este factor condicionante fuese el sistema de la HAD y se nos plantea entonces dos interrogantes:

19 ¿Hay un déficit cuantitativo de la hormona?

$2^{\circ}$ ¿Existe una alteración a nivel del efector?

Existiendo en el desnutrido una hipotonía manifiesta, sin alteraciones en el volumen de los líquidos corporales $(9,12,21,22)$ que sirvan de estímulo a la secreción y producción de HAD, es lógico suponer que los niveles de esta hormona, no sólo están disminuídos, sino que además exista un bloqueo en su síntesis y secreción. Este hecho, no demostrado en el desnutrido, podría ser incluído en la tendencia actual a considerar, que existan alteraciones en los niveles y funcionamientos del "Pool" hormonal en el desnutrido severo (9, $22,23,24,25,26$ ).

Otra posibilidad a considerar es que en presencia de niveles normales de la hormona, exista una respuesta hormonal pobre ante un estímulo agudo; esto se debería a una especie dc adaptación al estado de desnutrición prolongada (27).

Considerando ahora la disfunción, como producto de alteraciones del efector, deberíamos analizar el problema desde dos puntos de vista:

a) Alteraciones anatómicas del túbulo renal, y

b) Alteraciones bioquímicas.

Las primeras son inconstantes y generalmente octurren en la fase terminal de la desnutrición. Un estudio sobre este parámetro está siendo lle. vado a cabo por nosotros. Las alteraciones bioquímicás, cuyo estudio no está a nuestro alcance, 
deberían ser enfocadas hacia la determinación de AMP cíclico en la célula tubular y al estudio de los niveles de adenil-ciclasa y su especificidad hacia la HAD en las células del túbulo renal $(28,29)$ del lactante con desnutrición severa.

\section{RESUMEN}

Se analizó la capacidad renal de concentración urinaria en tres grupos de desnutridos; éstos grupos se establecieron en base a porcentajes de déficit de peso $(0-20,20-40$ y $40-60 \%)$. La prueha se realizó combinando deprivación acuosa de 12 hrs. de duración y la administración de Pitresina. Se observó que la incapacidad renal del desnutrido para concentrar la orina es proporcional al grado de su desnutrición.

\section{REFERENCJAS}

1.-Crawford D. 1. en "Bases Biológicas de la Práctica Pediátrica" de R. E. Cooke. Versión Española $1^{a}$ Edición. Salvat Editores. Barcelona, España.

2.-Dingham J. F. en "Harrison's principles of Internal Medicine" 6th Ed. Caledonia. The Colonnial Press. Inc. Cap. 88, Pág. 435, 1970.

3.- Hickford $M$. "Control of the secretion of antidiuretics Hormone". Pharmacol Rev. 4: 454, 1952.

4.-Mathie" $H$, en "Nefrología. Especialidades en Pediatría". Ed. Andrés Bello. Santiago, Chile. Vol. I, Pág. 161, 1968.

5.-Early L. E., Orieff J. "The mechanism of antidiureis associated with the administration of hidroclocotiazide to patient with Vassopresins Resistant Diabetes Insipidus". J. Clit. Invest. 41: 1988, 1962.

6.-Marshall P, F., Brovker G. "Deficient renal AMPc production in nephrogeric Diabetes Insipidus". J. Clin. End. Met. 35: 35, 1972.

7.- Winbers J. "Determination of renal concentration capacity in infants without renal dissase". Acta Pediat Scand. 48: 318, 1959.

8.-Gordillo P. G., Soto R. A., Metcoff J., López E. "Intracelular composition and homeostatics mecanism in levere infantil Malnutrition. III. Renal adjustement". Pedjatrics. 20: 303, 1957.

9.-Alleyne G. A. "The efects of severe Proteinecalorie malnutrition on the renal function of Jamaican Children". Pediatrics. 39: 400, 1967.
10.-Edozien J., Phyllips E. J "Partition of Urine Nitrogen in Kwashiorkkor" Nature (London) 191: $47,1961$.

11.-Dean $R . F$. "Nitrogenous constituents of urine in Kwasshiortor". Ped. Proc. (Suppl. 7) 20: 202, 1961.

12.-Wateriow J. C. "The partition of the Nitrogen in the urine of malnourished Jamaicun Infants". Amer. J. Clin. Nutr. 12: 235, 1963.

13.-Schwariz W. B., Relman A. S. "Metabolics and renal studies in cronic potassium deficiency resulting from overuse of laxative". J. Clin, Invest. 32: $228,1953$.

14.- Kerpet-froniss E., Romhaugi G., Gati $B$. "In. fluences of deppletion of potassium, of sodium or of water or function and structure of the kidney". Pediatrics. 26: 939, 1960.

15.-Holliday M. A., Segar W. E. Bright N. H. "The effect of potassium deficiency on the kidney". Pediatrics 26: 950, 1960.

16.- Levinciny N. G., Berliner R. W. "The role of urea in the concentrating mechanism". J. Clin. Invest. 38: $74 \mathrm{l}, 1959$.

17.-Gordilio P. G. en "Electrolitos en Pediatría. Fisiología y Clínica". 19 Edición. Impresiones Modernas, S. A. Méjico D. F. Pag. 97, 1971.

18.-Gordillo P. G. "Trastornos renales en niños con desnutrición avanzada". Bol. Med. Hosp. Inf. Mex. 21: 699, 1964.

19.-Vial S., Donoso P. "Efecto de la infusión continua de Vasopresina en la función renal de los niños normales y desnutridos". Revista Chilena de Pediatría. 1: 25, 1970.

20,-Edelman C, H. Barnett C. "Role of the kidney in water metabolism in young infants". J. Pediat. 56: 154,1960 .

21,- Metcoff H. E. "Renal Regulation of body Fluids". Ped. Clin. N. Amer. 11: 853, 1964.

22.-Prinstone B. L., Barbezat G., Hansen J. "Growth hormone and proteine caloric malnutrition". Lancet 2: $1333,1967$.

23.- Jame's W. P., Coore H. C. "Persisten impairment of insuline secretion and glucose tolerance after malnutrition". Amer. J. Clin. Nutr. 23: 386, 1970. 
24.- Baigs H. A., Edozien J. C. "Carbohidrate metaboJism in kwashiorkor". The Lancet 2: 662, 1965.

25.-Milner $R$. D. "Endocrine adaptation to malnutrition". Nutr. Rev. 30: 103, 1972.

26.--Grahan G. G., Baerth J., Closyssend G., Suskind $R$. "Thyroid hormonal studies in normal and severely malnourished infants and small children". J. Pediat. 83: 321, 1973.
27.-Rao J. K., Srikantia S., Gopolan C. "Plasma cortisol levels in proteins calorie malnutrition". Arch. Dis Child. 43: 365, 1968.

28.- Liddle G. W., Hardmast S. "Cyclic adenosine Monophosphate as mediator of hormone action". N. Engl. J. Med. 285: 560, 1971.

29.-Butcher $R$. W. "Role of cyclic AMP in Hormone action". N. Engl. J. Med. 279: 1378, 1968. 